\title{
A FURTHER GENERALIZATION OF HILBERT'S INEQUALITY
}

\section{HUGH L. MONTGOMERY AND JEFFREY D. VAALER}

§1. Introduction. Hilbert's inequality asserts that

$$
\left|\sum_{\substack{r, s \\ r \neq s}} \frac{a_{r} \bar{a}_{s}}{r-s}\right| \leqslant \pi \sum_{r}\left|a_{r}\right|^{2},
$$

for arbitrary complex numbers $a_{r}$. The constant $\pi$ was first obtained by Schur [5], and is best possible. Following a suggestion of Selberg, Montgomery and Vaughan [4] showed that

$$
\left|\sum_{\substack{r, s \\ r \neq s}} \frac{a_{r} \bar{a}_{s}}{\gamma_{r}-\gamma_{s}}\right| \leqslant \pi \delta^{-1} \sum_{r}\left|a_{r}\right|^{2},
$$

where the $\gamma_{r}$ are distinct real numbers and

$$
\delta=\min _{\substack{r . s \\ r \neq s}}\left|\gamma_{r}-\gamma_{s}\right|
$$

Still more generally, they showed also that

$$
\left|\sum_{\substack{r, s \\ r \neq s}} \frac{a_{r} \bar{a}_{s}}{\gamma_{r}-\gamma_{s}}\right|<\frac{3}{2} \pi \sum_{r}\left|a_{r}\right|^{2} \delta_{r}^{-1},
$$

where

$$
\delta_{r}=\min _{\substack{s \\ r \neq s}}\left|\gamma_{r}-\gamma_{s}\right|
$$

This latter inequality is considerably more delicate than (1), and it contains (1) apart from the larger constant. (It remains unknown whether (3) holds with the constant $\pi$.) We now formulate a still more general inequality which includes (3) apart from a further imprecision in the constant.

THEOREM. Let $\rho_{r}=\beta_{r}+i \gamma_{r}$ be complex numbers with $\beta_{r} \geqslant 0$, and let $\delta_{r}$ be given by (4). Then

$$
\left|\sum_{\substack{r, s \\ r \neq s}} \frac{a_{r} \bar{a}_{s}}{\rho_{r}+\bar{\rho}_{s}}\right|<84 \sum_{r}\left|a_{r}\right|^{2} \delta_{r}^{-1} .
$$


Since the possibility that this inequality might hold was proposed by researchers considering the distribution of zeros of $L$-functions on the one hand, and those considering questions of metric Diophantine approximation on the other, it may be hoped that this inequality will be of use in a variety of investigations. It is not difficult to construct examples from which it may be seen that the weaker hypotheses $\beta_{r} \geqslant 0,\left|\rho_{r}-\rho_{s}\right| \geqslant \delta_{r}$ do not imply an inequality of the above sort. On the other hand, Graham and Vaaler have established an inequality intermediate to (1) and (5) in which $\delta$ is given by (2) and all the $\beta$ 's are equal but with the best possible constant (see [1], equation (5.11)).

COROLlary. Under the above hypotheses, for any $U>0$,

$$
\int_{0}^{U}\left|\sum_{r=1}^{R} a_{r} e^{-\rho_{r} u}\right|^{2} d u=\sum_{r=1}^{R}\left|a_{r}\right|^{2} \frac{1-e^{2 \beta_{r} U}}{2 \beta_{r}}+168 \theta \sum_{r=1}^{R}\left|a_{r}\right|^{2} \delta_{r}^{-1}
$$

for some $\theta,-1 \leqslant \theta \leqslant 1$.

If $\beta_{r}>0$ for all $r$ then we can let $U \rightarrow \infty$ in the above.

\$2. Proof of the Theorem. Let $\rho_{r}^{\prime}=\delta_{r}+\rho_{r}$. We note that

$$
\frac{1}{\rho_{r}+\bar{\rho}_{s}}-\frac{1}{\rho_{r}^{\prime}+\bar{\rho}_{s}^{\prime}}=\left(\delta_{r}+\delta_{s}\right)\left(\rho_{r}+\bar{\rho}_{s}\right)^{-1}\left(\rho_{r}^{\prime}+\bar{\rho}_{s}^{\prime}\right)^{-1} \text {. }
$$

Since $\left|\rho_{r}+\bar{\rho}_{s}\right| \geqslant\left|\gamma_{r}-\gamma_{s}\right|$ and $\left|\rho_{r}^{\prime}+\bar{\rho}_{s}^{\prime}\right| \geqslant\left|\gamma_{r}-\gamma_{s}\right|$, it follows that

$$
\left|\sum_{\substack{r, s \\ r \neq s}} \frac{a_{r} \bar{a}_{s}}{\rho_{r}+\bar{\rho}_{s}}-\sum_{\substack{r, s \\ r \neq s}} \frac{a_{r} \bar{a}_{s}}{\rho_{r}^{\prime}+\bar{\rho}_{s}^{\prime}}\right| \leqslant \sum_{\substack{r, s \\ r \neq s}}\left(\delta_{r}+\delta_{s}\right) \frac{\left|a_{r} \bar{a}_{s}\right|}{\left(\gamma_{r}-\gamma_{s}\right)^{2}} .
$$

However, Montgomery and Vaughan [4] have shown (see the estimate of $T_{6}$ on pp. 80-81) that the expression on the right above is at most

$$
17 \sum_{r}\left|a_{r}\right|^{2} \delta_{r}^{-1}
$$

Here the constant 17 is not optimal, and it would be interesting to know what the best constant is. By taking $\gamma_{r}=r, a_{r}=1$ for all $r$, it is evident that the best constant is at least as large as $2 \pi^{2} / 3$.

In view of $(6)$, it is enough to show that

$$
\left|\sum_{\substack{r, s \\ r \neq s}} \frac{a_{r} \bar{a}_{s}}{\rho_{r}^{\prime}+\bar{\rho}_{s}^{\prime}}\right| \leqslant 67 \sum_{r}\left|a_{r}\right|^{2} \delta_{r}^{-1} .
$$

To simplify notation, from this point on we write $\rho_{r}$ for $\rho_{r}^{\prime}$, and assume that $\beta_{r} \geqslant \delta_{r}$. Clearly

$$
\sum_{r, s} \frac{a_{r} \bar{a}_{s}}{\rho_{r}+\bar{\rho}_{s}}=\int_{0}^{\infty}\left|\sum_{r=1}^{R} a_{r} e^{-\rho_{r} u}\right|^{2} d u
$$


Here the right-hand side is non-negative, and the terms $r=s$ on the left contribute an amount $\frac{1}{2} \sum_{r}\left|a_{r}\right|^{2} \beta_{r}^{-1} \leqslant \frac{1}{2} \sum_{r}\left|a_{r}\right|^{2} \delta_{r}^{-1}$. Hence

$$
\sum_{\substack{r, s \\ r \neq s}} \frac{a_{r} \bar{a}_{s}}{\rho_{r}+\bar{\rho}_{s}} \geqslant-\frac{1}{2} \sum_{r}\left|a_{r}\right|^{2} \delta_{1}^{-1},
$$

and to complete the proof it suffices to show that

$$
\int_{0}^{\infty}\left|\sum_{r=1}^{R} a_{r} e^{-\rho_{r} u}\right|^{2} d u \leqslant 67 \sum_{r}\left|a_{r}\right|^{2} \delta_{r}^{-1} .
$$

By the basic duality principle, as expressed for example by taking $p=q=2$ in Theorem 286 of Hardy, Littlewood and Pólya [2], the above is equivalent to the assertion that

$$
\sum_{r=1}^{R} \delta_{r}\left|\int_{0}^{\infty} f(u) e^{-\rho, u} d u\right|^{2} \leqslant 67 \int_{0}^{\infty}|f(u)|^{2} d u
$$

for all $f \in L_{[0, x)}^{2}$. Write $s=\sigma+i t$, and for $\sigma>0$ put

$$
F(s)=\int_{0}^{\infty} f(u) e^{-s u} d u .
$$

This function is analytic for $\sigma>0$, and is in the Hardy class $H^{2}$ on the half-plane $\sigma \geqslant 0$. From the basic properties of such functions, as discussed in Chapter 8 of Hoffman [3], for example, we know that $\lim _{\sigma \rightarrow 0^{+}} F(s)$ exists for almost all $t$; we call its value $F(i t)$. Moreover, $F(i t) \in L^{2}(\mathbb{R})$, and

$$
\int_{-\infty}^{\infty}|F(i t)|^{2} d t=2 \pi \int_{0}^{\infty}|f(u)|^{2} d u .
$$

For $\sigma>0$ we may express $F(s)$ in terms of $F(i t)$ by means of the Poisson kernel:

$$
F(s)=\frac{\sigma}{\pi} \int_{-\infty}^{\infty} \frac{F(i v)}{\sigma^{2}+(v-t)^{2}} d v .
$$

Let

$$
\theta(x)=\sup _{\substack{\xi \\ \xi \neq x}} \frac{1}{\xi-x} \int_{x}^{\xi}|F(i v)| d v
$$


be the Hardy-Littlewood maximal function of $F(i v)$. On integrating by parts in (10) we find that

$$
|F(s)| \leqslant \frac{2 \sigma}{\pi} \theta(x) \int_{-\infty}^{\infty} \frac{|v-x||v-t|}{\left(\sigma^{2}+(v-t)^{2}\right)^{2}} d v .
$$

As $|v-x||v-t| \leqslant|x-t||v-t|+(v-t)^{2}$, we find that the above is at most

$$
\theta(x)\left(\frac{2|t-x|}{\pi \sigma}+1\right)
$$

In this relation we take $s=\rho_{r}$, divide both sides by the expression in parentheses, square both sides, and integrate with respect to $x, \gamma_{r}-\delta_{r} / 2 \leqslant x \leqslant \gamma_{r}+\delta_{r} / 2$. This gives

$$
\delta_{r}\left|F\left(\rho_{r}\right)\right|^{2} \leqslant\left(1+\frac{\delta_{r}}{\pi \beta_{r}}\right) \int_{r_{r}-\delta_{r} / 2}^{\gamma_{r}+\delta_{r} / 2}|\theta(x)|^{2} d x .
$$

Here $\beta_{r} \geqslant \delta_{r}$ and the intervals of integration are disjoint for distinct $r$. Hence it follows that the left-hand side of $(8)$ is

$$
\sum_{r=1}^{R} \delta_{r}\left|F\left(\rho_{r}\right)\right|^{2} \leqslant\left(1+\frac{1}{\pi}\right) \int_{-\infty}^{\infty} \theta(x)^{2} d x .
$$

By the Hardy-Littlewood inequality (see p. 33 of Zygmund [6]), this latter integral is less than or equal to $8 \int_{-\infty}^{\infty}|F(i t)|^{2} d t$. Hence by (9) we see that (8) holds with constant $16 \pi(1+1 / \pi)=66 \cdot 265 \ldots<67$. This completes the proof.

To derive the Corollary it suffices to square out, integrate term-by-term, and apply the Theorem twice.

Acknowledgement. The research of the authors was supported in part by NSF grants DMS-9107605 and DMS-96-22556.

\section{References}

1. S. W. Graham and J. D. Vaaler. A class of extremal functions for the Fourier Transform. Trans. Amer. Math. Soc., 265 (1981), 283-302.

2. G. H. Hardy, J. E. Littlewood and G. Pólya. Inequalities (CUP, 1967).

3. K. Hoffman. Banach Spaces of Analytic Functions (Prentice-Hall, 1962).

4. H. L. Montgomery and R. C. Vaughan. Hilbert's Inequality. J. London Math. Soc. (2), 8 (1974), 73-82. 
5. I. Schur. Bemerkungen zur Theorie der beschränkten Bilinearformen mit unendlich vielen Veränderlichen. J. Reine Angew. Math., 140 (1911), 1-28.

6. A. Zygmund. Trigonometric Series Vols. I, II (CUP, 1968).

Professor Hugh L. Montgomery,

Department of Mathematics,

University of Michigan,

Ann Arbor, MI 48109-1109,

U.S.A.

Professor Jeffrey D. Vaaler,

Department of Mathematics,

The University of Texas,

Austin, TX 78712

U.S.A.
26D20: REAL FUNCTIONS; Inequalities; Other analytic inequalities.

Received on the 17th April, 1998. 